\title{
BMJ Open Impact of depression on clinical outcomes following percutaneous coronary intervention: a systematic review and meta-analysis
}

Wen Yi Zhang, Nan Nan, Xian Tao Song, Jin Fan Tian, Xue Yao Yang

To cite: Zhang WY, Nan N, Song XT, et al. Impact of depression on clinical outcomes following percutaneous coronary intervention: a systematic review and meta-analysis. BMJ Open 2019;9:e026445. doi:10.1136/ bmjopen-2018-026445

- Prepublication history and additional material for this paper are available online. To view these files, please visit the journal online (http://dx.doi org/10.1136/bmjopen-2018026445).

WYZ and NN are joint first authors.

Received 08 September 2018 Revised 21 March 2019 Accepted 05 July 2019

\section{Check for updates}

(C) Author(s) (or their employer(s)) 2019. Re-use permitted under CC BY-NC. No commercial re-use. See rights and permissions. Published by BMJ.

Department of Cardiology, Beijing An Zhen Hospital, Capital Medical University, Beijing Institute of Heart, Lung and Blood Vessel Diseases, Beijing, China

Correspondence to Professor Xian Tao Song; songxianta00929@qq.com

\section{ABSTRACT}

Objectives The objective of this meta-analysis was to assess whether depression in percutaneous coronary intervention $(\mathrm{PCl})$ patients is associated with higher risk of adverse outcomes.

Design Systematic review and meta-analysis. Methods EMBASE, PubMed, CINAHL and PsycINF0 were searched as data sources. We selected prospective cohort studies evaluating the relationship between depression and any adverse medical outcome, including all-cause mortality, cardiac mortality and non-fatal events, from inception to 28 February 2019. Two reviewers independently extracted information and calculated the risk of cardiovascular events in patients with preoperative or postoperative depression compared with non-depressed patients.

Results Eight studies ( $n=3297$ ) met our inclusion criteria. Most studies found a positive association between depression and adverse cardiovascular outcomes. Metaanalysis yielded an aggregate risk ratio of $1.57(95 \% \mathrm{Cl}$ 1.28 to $1.92, p<0.0001$ ) for the magnitude of the relation between depression and adverse outcomes.

Conclusions Our systematic review and meta-analysis suggests that depression is associated with an increased risk of worse clinical outcome or mortality in patients undergoing $\mathrm{PCl}$. Assessment time and length of follow-up do not have a significant effect on this conclusion.

\section{INTRODUCTION}

Depression and coronary artery disease (CAD) are highly comorbid conditions with estimates of comorbidity from $20 \%$ to $50 \% .^{1-3}$ Patients with a combination of depression and CAD are at increased risk for negative cardiac outcomes. Prior meta-analyses have demonstrated that depression is associated with a twofold to fourfold increase in the risk of future major adverse cardiovascular events in patients with postmyocardial infarction (MI), and this relationship has remained stable despite improvements in diagnosis and treatment. ${ }^{45}$ The adverse effects of depression are also observed in patients admitted with unstable angina. ${ }^{6}$

\section{Strengths and limitations of this study}

- This study emphasises the growing recognition that depression is a major risk factor of cardiovascular disease.

- Depression needs to be fully considered when assessing the prognosis of percutaneous coronary intervention patients.

- The quality of the included studies was relatively high.

- Further analyses were difficult to conduct due to the limited number of studies.

Percutaneous coronary intervention (PCI) has become a common revascularisation procedure with demonstrated safety and efficacy. Successful PCI significantly reduces the rate of death or revascularisation, and improves quality of life. ${ }^{78}$ Nonetheless, major adverse cardiac events (MACE), such as mortality, non-fatal MI and repeat PCI, still remain a problem. According to several studies, about one-fifth of patients experience depression before PCI, an even greater proportion of patients are depressed after the procedure. $^{9-11}$ Besides well-known factors, psychological factors such as depression have been demonstrated to predict adverse cardiac events after PCI by multiple studies. ${ }^{12-15}$ However, not all the studies have suggested that depression was associated with poor prognosis following PCI. ${ }^{16}$ Meyer et $a l^{16}$ reported depressive symptoms were linked to mortality during 2 years' follow-up, but the relationship disappeared 3 years later. Moreover, de Jager ${ }^{17}$ and colleagues found the predictive value of depression differs between angina pectoris (stable angina, $\mathrm{SA}$ ) and acute coronary syndrome (ACS) cohorts. Furthermore, levels of depression tend to change over time and questions remain about when to assess depression. Considering these problems, we performed a systematic review and 


\section{Box 1 Search strategy in PubMed}

1. depression [Mesh]

2. depression*[Title/Abstract]

3. depressive disorder*[Title/Abstract]

4. depressive $\operatorname{mood}^{*}[$ [itle/Abstract]

5. dysthymia [Title/Abstract]

6. No 1 OR No 2 OR No 3 OR No 4 OR № 5

7. percutaneous coronary interventions [Mesh]

8. percutaneous coronary intervention*[Title/Abstract]

9. $\mathrm{PCl}$ [Title/Abstract]

10. Coronary Balloon Angioplasty [Title/Abstract]

11. angioplasty [Title/Abstract]

12. № 7 OR No 8 OR No 9 OR No 10 OR No 11

13. No 6 AND № 12

meta-analysis to determine the prospective relationship between depression and adverse clinical outcomes after PCI.

\section{METHODS}

A systematic review and meta-analysis was performed following Preferred Reporting Items for Systematic Reviews and Meta-Analyses guidelines ${ }^{18}$ and an a priori study protocol.

\section{Search strategy and selection criteria}

Two authors (WYZ and NN) independently searched the literature in EMBASE, PubMed, CINAHL and PsycINFO databases without language restriction from inception to 28 February 2019. The search strategy contained keywords related to the population of interest (patients with CAD receiving PCI) and keywords related to depression. In PubMed, we used the combination of Medical Subject Headings (MeSH terms) and relevant free-text terms to identify the keywords for each topic. The set operator AND was used to form a complementary search strategy (see box 1).

The search terms from PubMed were adapted to the corresponding vocabulary of EMBASE, CINAHL and PsycINFO databases.

\section{Selection and exclusion criteria}

Studies investigating the association between depression and prognosis in patients receiving PCI were retrieved for review. Inclusion criteria were as follows: (1) prospective design, (2) patients diagnosed with $\mathrm{CAD}$ and receiving coronary stent implantation, (3) using established assessment instruments or structured clinical interviews to define major depression or depressive symptoms, and (4) reporting an endpoint of all-cause mortality, cardiac mortality, rehospitalisation or MACEs.

The exclusion criteria were as follows: (1) case report, animal research, review article or expert opinion, (2) depression not considered as a predictor, (3) unrelated to the search terms, (4) participants without PCI, and (5) data were not reported. For multiple publications from the same cohort, ${ }^{12}{ }^{19-22}$ we chose the latest or most complete study for assessment. ${ }^{12}$

\section{Data extraction}

Two authors independently (JFT and XYY) read the abstract and title of every record identified by the search. Potentially eligible studies were reviewed in detail. Differences in opinion were resolved by consensus. Given that a variety of factors may influence outcome, the following data were extracted from the final eight studies by use of separate spreadsheets: first author's name, study design, ethnicity of the study population, diagnosis of CAD, depression measurement, timing of assessment, outcome definition, length of follow-up, sample size and number of patients in the depressed and non-depressed groups.

\section{Quality assessment}

We used the Newcastle-Ottawa Scale ${ }^{23}$ for quality assessment of included studies. The Newcastle-Ottawa Scale grades three domains: the selection of participants, comparability of the groups and assessment of outcome. ${ }^{23}$ A study can be awarded a maximum of 9 stars for quality. Each study was assessed independently by two investigators. The results are reported in table 1, online supplementary table S1.

\section{Data synthesis and statistical analysis}

Review Manager (RevMan) (V.5.3, The Cochrane Collaboration, 2014) served as the statistical platform for data management and statistical analyses. Dichotomous frequency data were extracted from each study. Risk ratios (RR) were calculated from pooled data comparing depressed and non-depressed groups for the likelihood of adverse cardiovascular events. We calculated a pooled RR and $95 \% \mathrm{CI}$ in the random effects model to account for possible methodological and clinical heterogeneity.

\section{Heterogeneity analysis}

The statistical heterogeneity among studies was evaluated using the Cochran's $Q$ test, $I^{2}$ statistic and $\mathrm{df}$. The $Q$ test was used to estimate test heterogeneity among trials. The $Q$ value can be used to derive the $\mathrm{I}^{2}$ value, which is the proportion (\%) of variance in a pooled effect size due to heterogeneity rather than chance. Based on $\mathrm{I}^{2}$, heterogeneity was rated as low $\left(\mathrm{I}^{2}<50 \%\right)$, moderate $(50 \%-75 \%)$ or high $(>75 \%){ }^{24}$

\section{Publication bias}

Publication bias was assessed by means of Egger's regression asymmetry test ${ }^{25}$ and Begg's test. ${ }^{26}$ We used the funnel plot to examine whether sample sizes influenced the results of the meta-analysis.

\section{Sensitivity analysis and subgroup analysis}

To further explore the sources of heterogeneity, we conducted a sensitivity analysis by omitting one study at a time and calculating the pooled effect size, $95 \% \mathrm{CI}$ and heterogeneity of the remaining studies (table 2 ). 


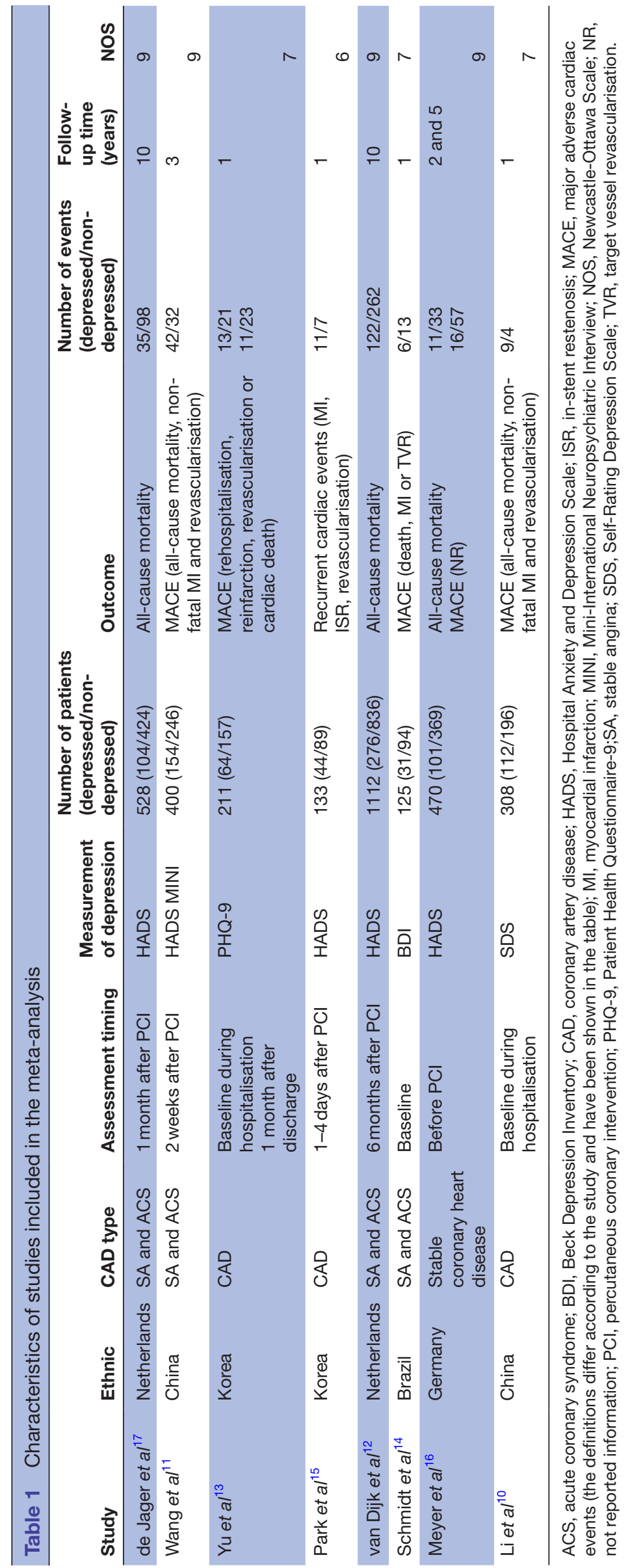


Table 2 Sensitivity analyses: results when given named study is omitted

\begin{tabular}{|c|c|c|c|}
\hline Study omitted & Risk ratio $(95 \% \mathrm{Cl})$ & Heterogeneity $\left(\left(^{2}\right)\right.$ & $P$ value \\
\hline de Jager et $a l^{17}$ & 1.63 (1.25 to 2.13$)$ & $44 \%$ & $<0.001$ \\
\hline Wang et al ${ }^{11}$ & 1.47 (1.21 to 1.79$)$ & $24 \%$ & $<0.001$ \\
\hline Park et al ${ }^{15}$ & 1.50 (1.25 to 1.79$)$ & $24 \%$ & $<0.001$ \\
\hline van Dijk et $a l^{12}$ & 1.67 (1.26 to 2.21$)$ & $38 \%$ & $<0.001$ \\
\hline Meyer et $a l^{16}$ & 1.64 (1.34 to 2.02 ) & $31 \%$ & $<0.001$ \\
\hline Li et $a l^{10}$ & 1.51 (1.26 to 1.81$)$ & $25 \%$ & $<0.001$ \\
\hline
\end{tabular}

We conducted subgroup analyses to assess the possible moderator effects for the association of depression with prognosis after PCI. These moderator effects included the time point for evaluating depression, the type of outcome and length of follow-up.

\section{Patient and public involvement}

No patients were involved in the design, recruitment or conduct of the study. There are no plans to disseminate the results of the research to the patient community included in the trials of the review.

\section{RESULTS}

Study selection and description

A flow diagram of the literature search is illustrated in figure 1. A total of 2000 records were identified through the literature search, with 1826 articles remaining when duplicates were removed. These articles were evaluated in detail. Ultimately, eight studies met the inclusion criteria and were included in the meta-analysis. ${ }^{10-17}$ The included studies were published between 2011 and 2018, and had follow-up periods ranging from 1 to 10 years. Sample size ranged from 125 to 1112 , and the eight studies included

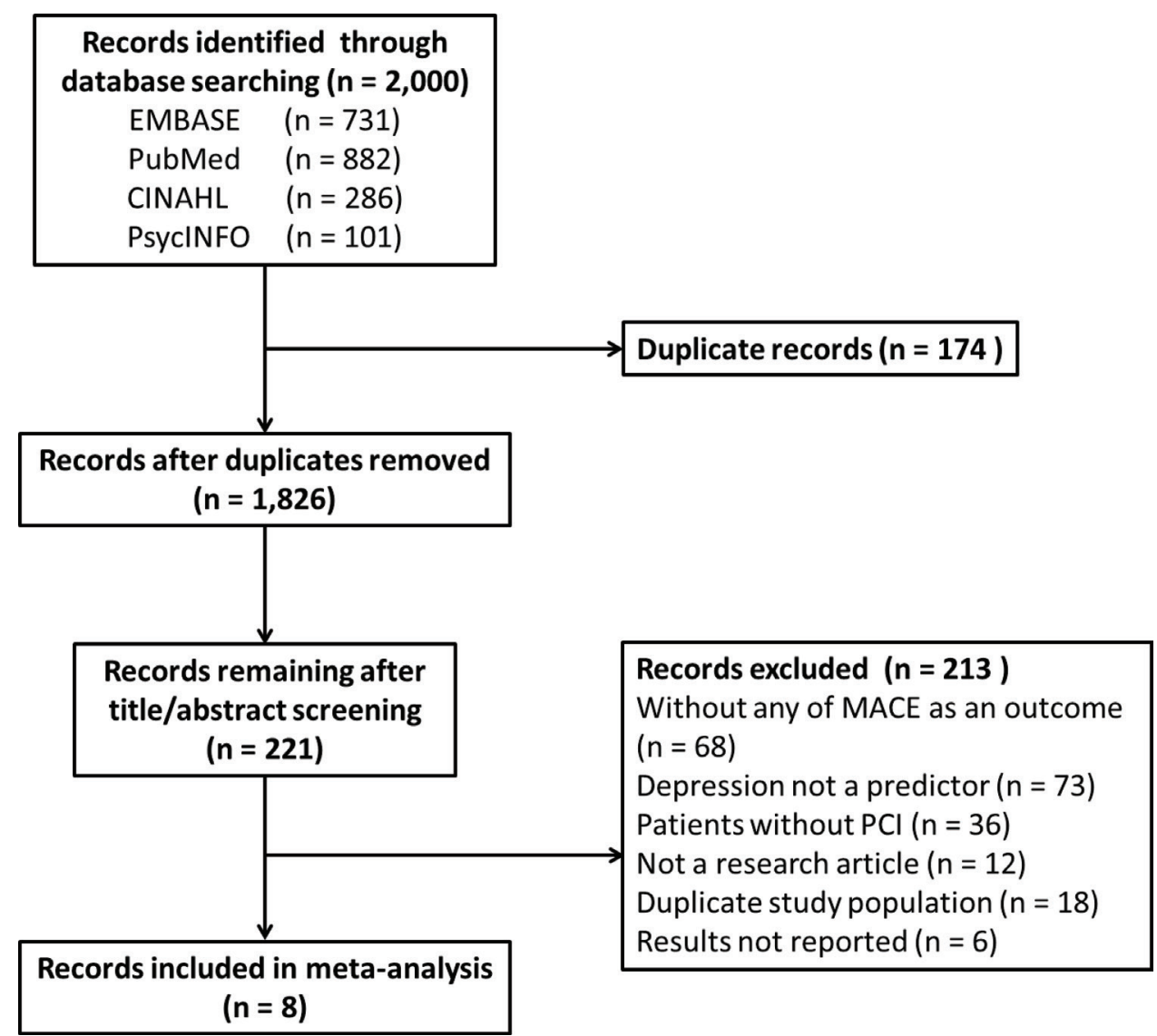

Figure 1 Preferred Reporting Items for Systematic Reviews and Meta-Analyses (PRISMA) flow chart for systematic review of depression and cardiovascular events following percutaneous coronary intervention (PCI). MACE, major adverse cardiac event. 
a total of 3297 participants from the Netherlands, China, Korea, Brazil and Germany. The quality of the studies was good, with 4 of $8(50 \%)$ studies rated 9 stars on the Newcastle-Ottawa Scale.

Of the identified studies five measured depression only once, varying from before the procedure during hospitalisation to 6 months after PCI. Three of the studies assessed depression twice. The research by Yu et al [13] measured depression at baseline during hospitalisation and 1 month after discharge. Wang et al [11] and Li et al [10] assessed depression both before and after the PCI. These two studies suggested depression was present in nearly $40 \%$ of the postoperative patients, increasing more than 10 percentage points than that before the operation.

Four of the eight studies defined depression according to the Hospital Anxiety and Depression Scale (HADS). ${ }^{27}$ The level of depression was considered clinically relevant at a cut-off HADS score of 8 . Wang et al [11] used a combination of HADS and the Mini-International Neuropsychiatric Interview to identify patients with depression. Yu $e t$ al [13] used the 9-Question Primary Care Evaluation of Mental Disorders Brief Patient Health Questionnaire-9 to define depression (non-depressed $\leq 4$, depressed 5-27). Schmidt et al [14] identified patients with depression using the Beck Depression Inventory with 20 points as the cut-off value. The study by Li et al [10] defined depression according to the Zung Self-Rating Depression Scale.

Two studies reported all-cause mortality, four studies reported adverse cardiac events as an outcome and two studies reported both all-cause mortality and adverse cardiac events separately. MACE was defined according to the individual study criteria and included all-cause mortality, non-fatal MI, revascularisation (as evidenced by repeated PCI, target vessel revascularisation, target lesion revascularisation or coronary artery bypass graft (CABG)) and rehospitalisation with cardiac death. Specific characteristics of the included studies are summarised in table 1.

\section{Meta-analysis results}

Effect of depression assessed at any time on composite outcome For the pooled sample (eight studies), depression assessed at any time period resulted in a significant increase in the incidence of cardiac events ( $\mathrm{RR}=1.57,95 \%$ CI 1.28 to 1.92 ) in the random effects model, indicating that depressed individuals have a $57 \%$ greater risk of poor outcome than non-depressed patients. The overall results are displayed in figure 2.

\section{Depression and all-cause mortality as an outcome}

As shown in figure 3 , in the subanalysis of four studies that included all-cause mortality as an outcome, depression was associated with a significantly higher risk of death after PCI ( $R R=1.43$, 95\% CI 1.24 to 1.65$)$.

\section{Sensitivity analysis}

To determine the reliability of the results, sensitivity analyses were performed by omitting one study at a time. No individual study had a substantial impact on the pooled effect size and heterogeneity. The RR changed slightly only when excluding the study by van Dijk et $\mathrm{al}^{12}$ with the largest number of participants ( $\mathrm{RR}=1.67,95 \%$ CI 1.26 to 2.21) (see table 2).

\section{Publication bias}

The funnel plot demonstrated slight asymmetry (figure 4), suggesting that there may be unpublished studies which have found no relationship between depression and clinical outcomes. However, neither Egger's test nor Begg's test revealed evidence of publication bias $(p>0.1)$, although these results should be interpreted with caution due to the limited number of included studies.

\section{Subgroup analysis}

Subgroup analysis by depression assessment time

The pooled RR was also calculated for studies assessing depression during hospitalisation and for those measuring depression 2 weeks or more after PCI. For studies with depression assessed in hospital, RR=1.71 (95\% CI 1.06 to 2.73) and for those with depression evaluated 2 weeks or more after PCI, RR=1.65 (95\% CI 1.30 to 2.08) (figure 5).

\section{Subgroup analysis by follow-up time}

We used 1year as the cut-off for distinguishing shortterm from long-term follow-up to evaluate whether the prognostic value of depression for predicting adverse outcomes was temporally limited. The pooled RR for studies with follow-up time less than or equal to 1 year

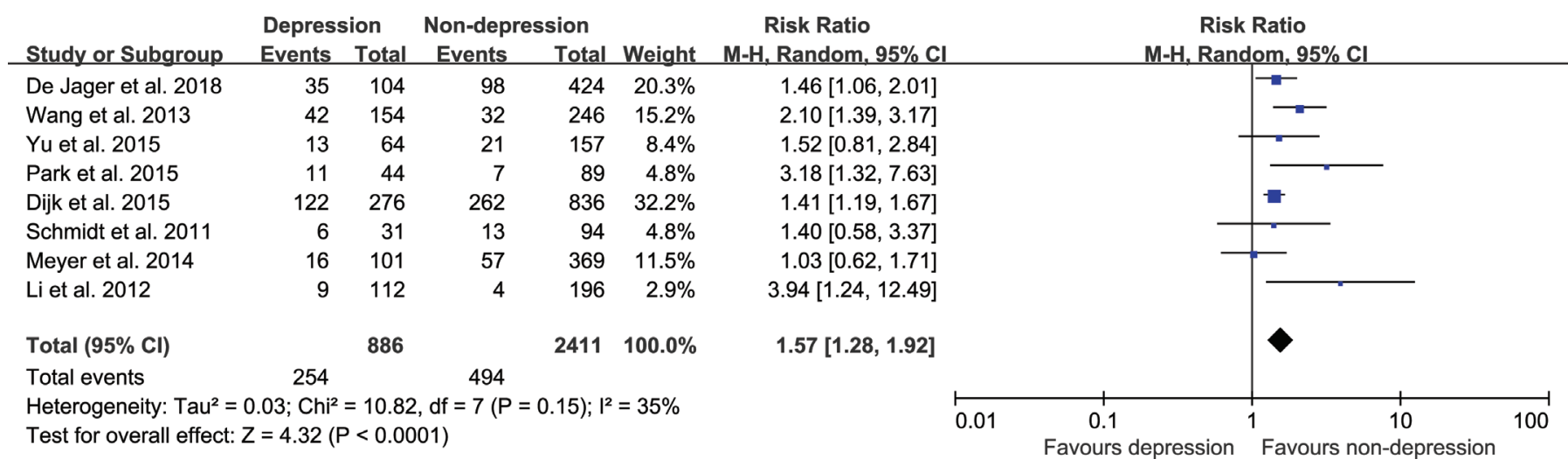

Figure 2 Forest plot of depression and a composite outcome following percutaneous coronary intervention (PCl). 


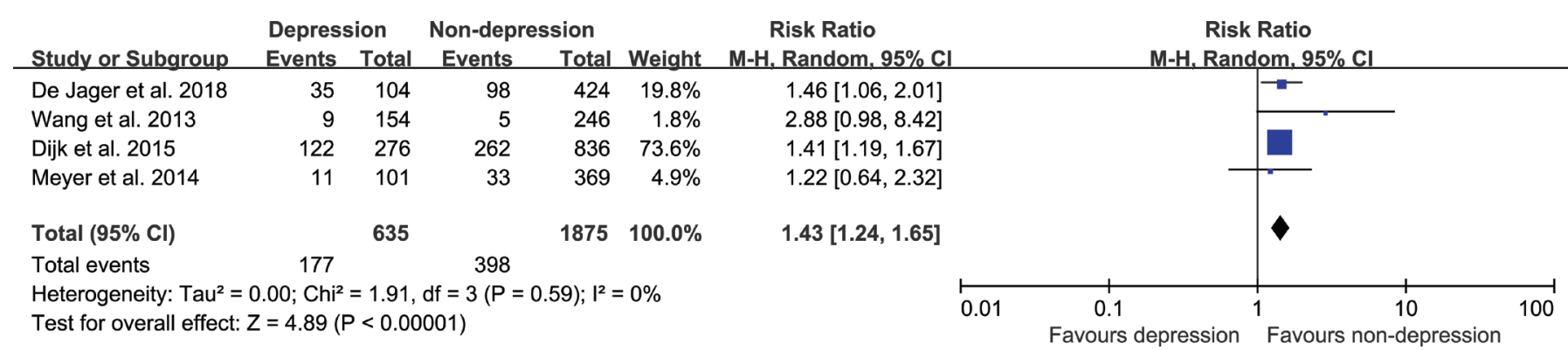

Figure 3 Forest plot of depression and all-cause mortality as an outcome following percutaneous coronary intervention (PCI).

was 2.04 (95\% CI 1.27 to 3.28), whereas the increase in risk became less pronounced when examining composite outcomes with longer follow-up times $(\mathrm{RR}=1.46,95 \% \mathrm{CI}$ 1.19 to 1.80 ) (figure 6).

\section{Heterogeneity}

Heterogeneity across studies was determined to test the appropriateness of combining studies. Slight heterogeneity was apparent among all included studies $\left(\mathrm{I}^{2}=35 \%\right.$, $\mathrm{p}$ for heterogeneity $=0.15)$. However, heterogeneity remained low $\left(\mathrm{I}^{2}=0 \%-49 \%\right)$ in all subgroups, indicating relative consistency across studies.

\section{DISCUSSION}

By combining the results from eight prospective observational cohort studies with 3297 participants, this meta-analysis presents evidence that symptoms of depression are associated with a $57 \%$ higher risk of adverse clinical outcome and a $43 \%$ higher risk of mortality in patients undergoing PCI. Sensitivity and subgroup analyses suggested that this relationship was not markedly affected by the timing of depression assessment, length of patient follow-up or type of outcome. Ultimately, the results of this study emphasise the growing recognition that depression is a major risk factor for poor outcomes in patients with coronary heart disease (CHD).

The relationship between CHD and depression has been widely examined. Meta-analyses have demonstrated

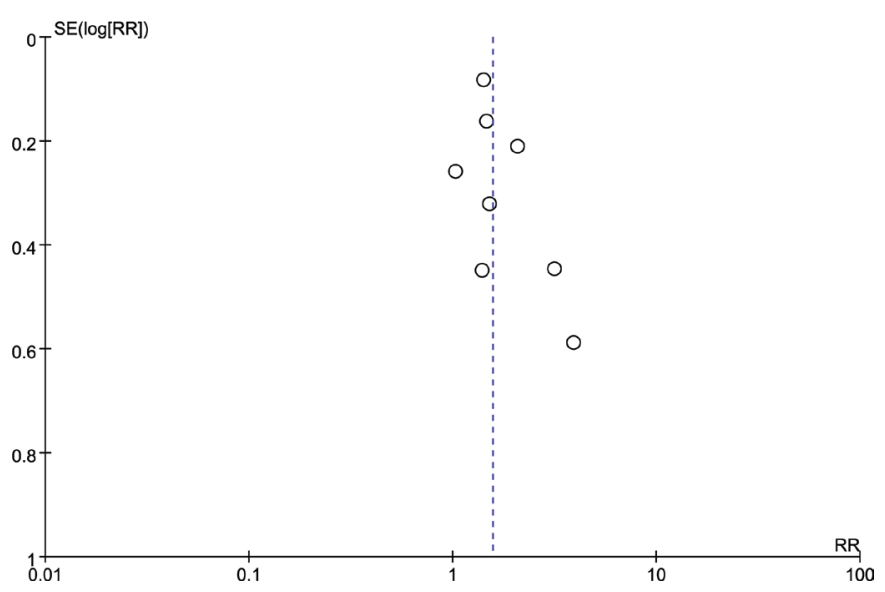

Figure 4 Funnel plot of depression and a composite outcome following percutaneous coronary intervention (PCI). $\mathrm{RR}$, risk ratio. that depressive symptoms have an unfavourable impact on mortality and cardiovascular events in CHD or patients with post-MI. ${ }^{52}$ However, little is known about the impact of depression on prognosis in PCI patients. We found that patients with depression exhibited a significantly increased risk for the primary endpoint of MACE and for the secondary endpoint of death. The results of this meta-analysis are in concordance with prior findings focusing on other CHD populations.

While a positive correlation between depression symptoms and adverse cardiac outcomes was found in our research, careful consideration should be given to different methods of depression assessment. In the majority of included articles, depression was defined based on scores of a self-report screening instrument, for example, the HADS, rather than structured or semistructured diagnostic interviews. Although several screening instruments have shown high sensitivity or specificity for patients with $\mathrm{CAD}$ and were used more often by physicians in general hospitals to assess depression, ${ }^{29-31}$ no consensus has yet been reached on the optimal screening tool for use in identifying depression in patients with CHD. ${ }^{32}$ Since the word 'depression' may include different meanings ranging from transient negative emotions through to serious clinical symptoms, more caution needs to be taken when investigating the prognostic value of depression in further studies.

Considering that several previous studies failed to demonstrate a negative impact of depression on outcome during long-term follow-up, ${ }^{16} 17$ we conducted a subgroup analysis according to follow-up time. The predictive value of depression was significant in both groups but less pronounced in the long-time follow-up group ( $R R=1.46 \mathrm{vs}$ $\mathrm{RR}=2.04$ for short-term follow-up). Whether depression still has a marked effect on the long-term prognosis of PCI requires further study.

Although the prevalence of depression after PCI is approximately $20 \%-30 \%$, the symptoms are likely to abate during recovery. Previous studies have found that PCI contributes to a higher risk of developing depressive symptoms in patients with CHD during hospitalisation. ${ }^{933}$ Therefore, depression measured too close to the point of interventional treatment may reflect a transient stress response and (or) worsening of physical symptoms. A meta-analysis by Ravven $e t a p^{4}$ showed that the risk of depression decreased throughout the long-term 
Group A: Depression assessed baseline during hospitalisation

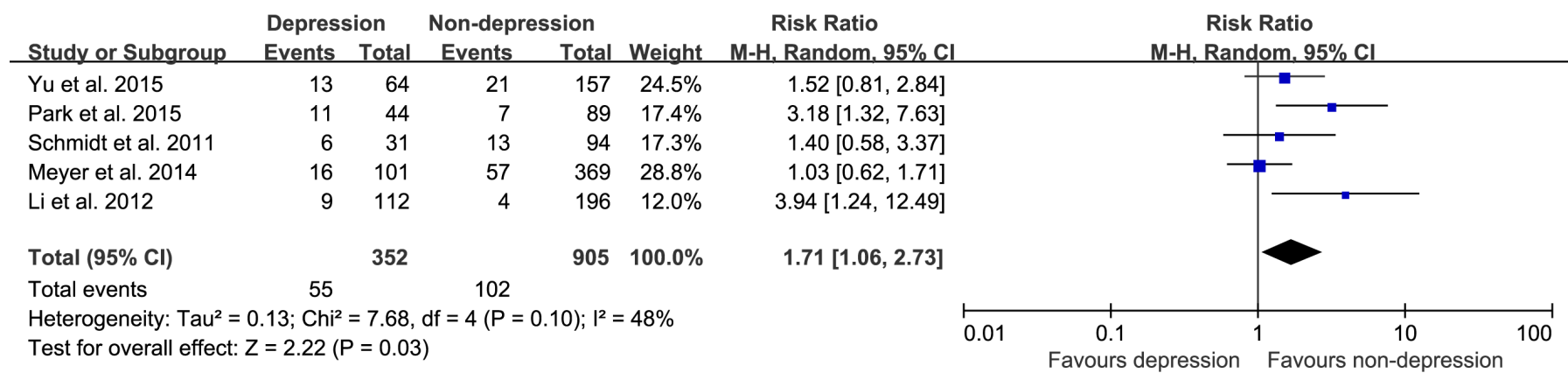

\section{Group B: Depression assessed two weeks or more after PCI}

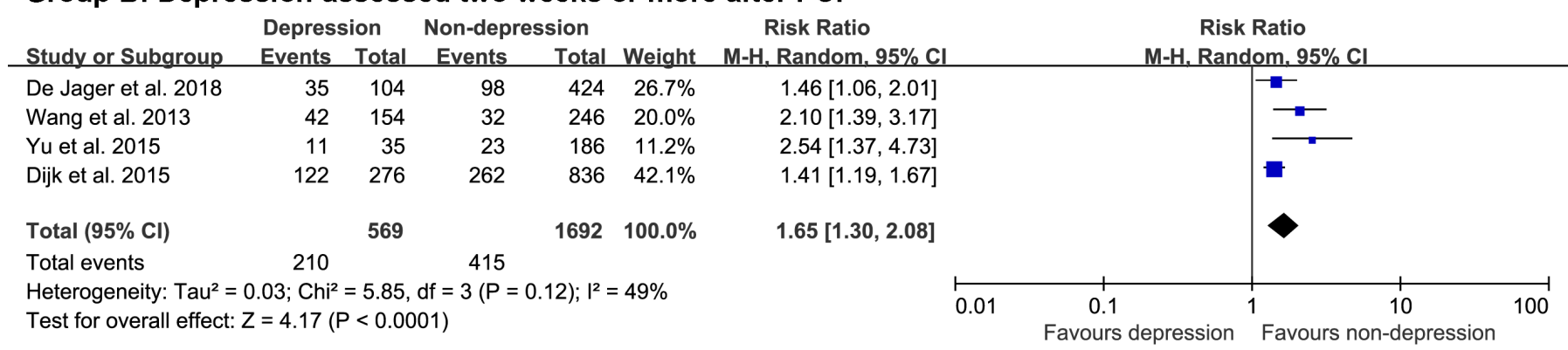

Figure 5 (A, B) The influence of different evaluation time of depression on the risk of adverse cardiac events. $\mathrm{PCl}$, percutaneous coronary intervention.

post-CABG period and that measurements taken in the 2 weeks after the operation may reflect the known consequences of surgery rather than a mood disorder. For these reasons, we also performed a subanalysis to determine the potential impact of evaluation time on the relationship between depression and prognosis. In this case, depression increased the risk of poor outcome whether assessed during hospitalisation ( $\mathrm{RR}=1.71,95 \%$ CI 1.06 to 2.73 ) or $>2$ weeks after PCI ( $R R=1.65,95 \%$ CI 1.30 to 2.08), indicating that the evaluation time has little influence on the adverse effects of depression. This result is in line with another recent finding that depression diagnosis at any time following CAD diagnosis was associated with an increased risk of death. ${ }^{35}$ The timing of depression

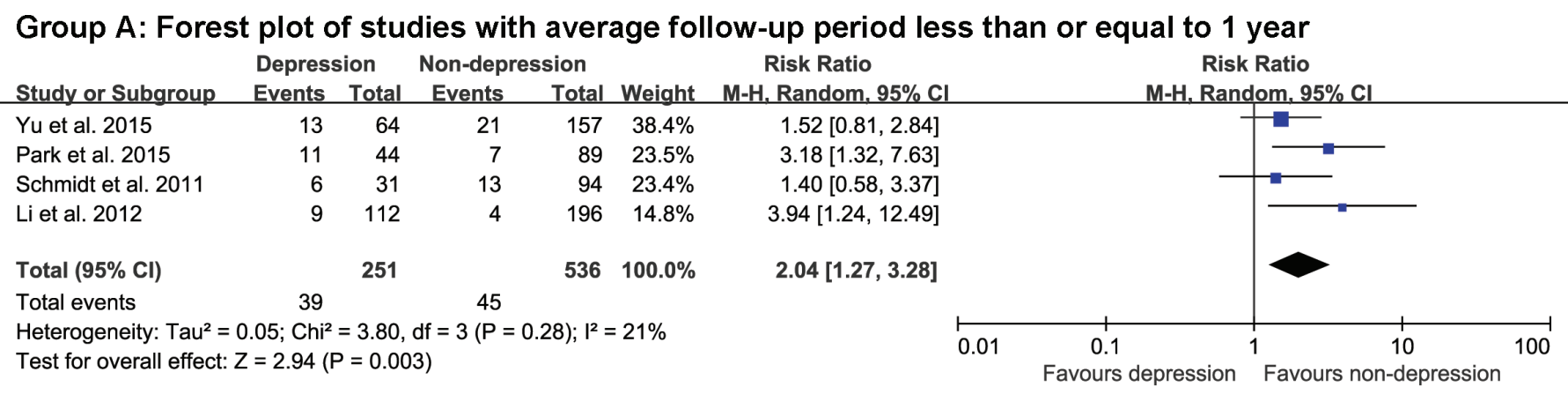

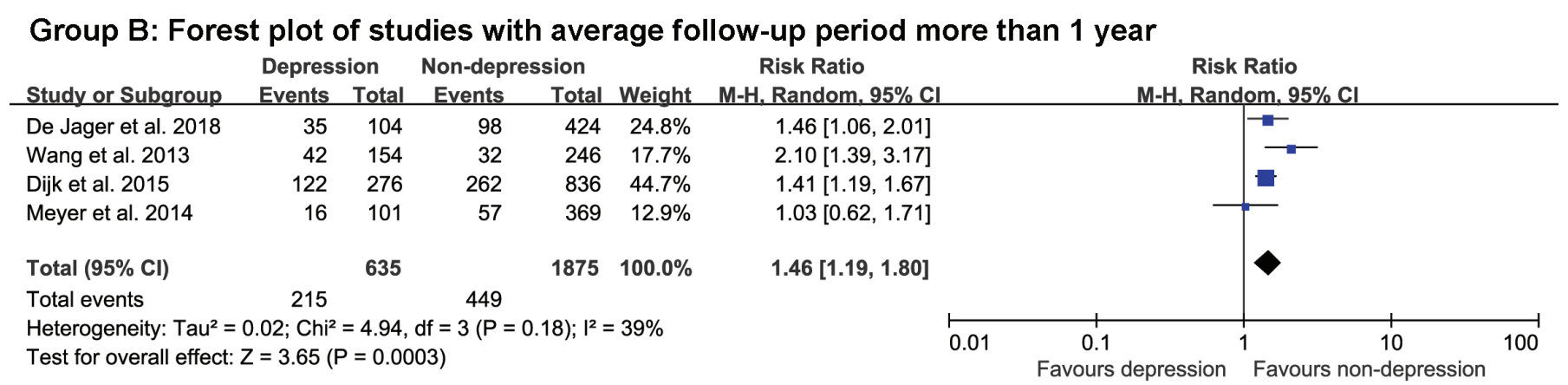

Figure 6 Relationship between depression and (A) short-term or $(B)$ long-term prognosis after percutaneous coronary intervention $(\mathrm{PCl})$. 
measures and the clinical significance of tests conducted at different times before or after PCI should be carefully considered in future studies.

The results of this meta-analysis have some limitations. Few studies have examined the relationship between depression and adverse clinical outcomes following PCI; thus, only eight studies were suitable for inclusion. Studies included in the meta-analysis were observational and were subject to patient selection bias, lack of independent events adjudication and heterogeneity in exposure definitions. Depression was analysed as a dichotomous variable, limiting examination of relationships between depressive symptom severity and clinical outcomes in patients treated by PCI. In addition, although some studies investigated the prognostic value of depression in different indication groups (SA and ACS), most did not report the results separately; therefore, we were unable to analyse depression risk by patient indications for PCI. Finally, because of the small number of studies in this field, we could not conduct further subgroup analyses, and this may have certain impact on the accuracy of our research.

\section{CONCLUSIONS}

This meta-analysis suggests that depression is associated with increased mortality and a greater risk of adverse clinical outcome after PCI. The risk appears to be stable whether depressive symptoms are measured in hospital or following treatment. The identification of depression in PCI patients is critical in view of its negative effect on postoperative recovery, morbidity and mortality.

Contributors All authors contributed to conception and design or analysis and interpretation of data. WYZ and NN performed the experiments. XTS, JFT and XYY analysed the data. WYZ, NN and JFT drafted the manuscript. XTS and XYY helped revise it critically for important intellectual content. All authors approved the final submission of the manuscript.

Funding The China Cardiovascular Disease Alliance VG Youth Fund Project (Grant No 2017- CCA-VG-007) and the Beijing Lab for Cardiovascular Precision Medicine funded the study.

Competing interests None declared.

Patient consent for publication Obtained.

Provenance and peer review Not commissioned; externally peer reviewed.

Data availability statement No data are available.

Open access This is an open access article distributed in accordance with the Creative Commons Attribution Non Commercial (CC BY-NC 4.0) license, which permits others to distribute, remix, adapt, build upon this work non-commercially, and license their derivative works on different terms, provided the original work is properly cited, appropriate credit is given, any changes made indicated, and the use is non-commercial. See: http://creativecommons.org/licenses/by-nc/4.0/.

\section{REFERENCES}

1. Lane D, Carroll D, Ring C, et al. The prevalence and persistence of depression and anxiety following myocardial infarction. $\mathrm{Br} \mathrm{J}$ Health Psychol 2002;7:11-21.

2. Thombs BD, Bass EB, Ford DE, et al. Prevalence of depression in survivors of acute myocardial infarction. $J$ Gen Intern Med 2006;21:30-8.

3. Gonzalez MB, Snyderman TB, Colket JT, et al. Depression in patients with coronary artery disease. Depression 1996;4:57-62.
4. van Melle JP, de Jonge P, Spijkerman TA, et al. Prognostic association of depression following myocardial infarction with mortality and cardiovascular events: a meta-analysis. Psychosom Med 2004;66:814-22.

5. Meijer A, Conradi HJ, Bos EH, et al. Prognostic association of depression following myocardial infarction with mortality and cardiovascular events: a meta-analysis of 25 years of research. Gen Hosp Psychiatry 2011;33:203-16.

6. Lespérance F, Frasure-Smith N, Juneau M, et al. Depression and 1-year prognosis in unstable angina. Arch Intern Med 2000;160:1354-60.

7. Xaplanteris P, Fournier S, Pijls NHJ, et al. Five-Year outcomes with $\mathrm{PCl}$ guided by fractional flow reserve. N Engl J Med 2018;379:250-9.

8. Baron SJ, Chinnakondepalli K, Magnuson EA, et al. Quality-ofLife After Everolimus-Eluting Stents or Bypass Surgery for LeftMain Disease: Results From the EXCEL Trial. J Am Coll Cardiol 2017;70:3113-22.

9. Park M-W, Kim J-H, Her S-H, et al. Effects of percutaneous coronary intervention on depressive symptoms in chronic stable angina patients. Psychiatry Investig 2012;9:252.

10. Li X-ming, Li T-ting, Cong H-liang, et al. [Impact of depression on prognosis of patients with coronary heart disease undergoing revascularization]. Zhonghua Xin Xue Guan Bing Za Zhi 2012;40:99-103.

11. Wang ZJ, Guo M, Si TM, et al. Association of depression with adverse cardiovascular events after percutaneous coronary intervention. Coron Artery Dis 2013;24:589-95.

12. van Dijk MR, Utens EMWJ, Dulfer K, et al. Depression and anxiety symptoms as predictors of mortality in $\mathrm{PCl}$ patients at 10 years of follow-up. Eur J Prev Cardiol 2016;23:552-8.

13. Yu HY, Park Y-S, Son Y-J. Combined effect of left ventricular ejection fraction and post-cardiac depressive symptoms on major adverse cardiac events after successful primary percutaneous coronary intervention: a 12-month follow-up. Eur J Cardiovasc Nurs 2017;16:37-45.

14. Schmidt MM, Quadros AS, Abelin AP, et al. Psychological characteristics of patients undergoing percutaneous coronary interventions. Arq Bras Cardiol 2011;97:331-7.

15. Park J-H, Tahk S-J, Bae SH. Depression and anxiety as predictors of recurrent cardiac events 12 months after percutaneous coronary interventions. J Cardiovasc Nurs 2015;30:351-9.

16. Meyer T, Hussein S, Lange HW, et al. Transient impact of baseline depression on mortality in patients with stable coronary heart disease during long-term follow-up. Clin Res Cardiol 2014;103:389-95.

17. de Jager TAJ, Dulfer K, Radhoe S, et al. Predictive value of depression and anxiety for long-term mortality: differences in outcome between acute coronary syndrome and stable angina pectoris. Int J Cardiol 2018;250:43-8.

18. Moher D, Liberati A, Tetzlaff J, et al. Preferred reporting items for systematic reviews and meta-analyses: the PRISMA statement. PLoS Med 2009;6:e1000097.

19. Pedersen SS, Denollet J, de Jonge P, et al. Brief depression screening with the $\mathrm{PHQ}-2$ associated with prognosis following percutaneous coronary intervention with paclitaxel-eluting stenting. $J$ Gen Intern Med 2009;24:1037-42.

20. Damen NL, Versteeg H, Boersma E, et al. Depression is independently associated with 7-year mortality in patients treated with percutaneous coronary intervention: results from the research registry. Int J Cardiol 2013;167:2496-501.

21. Pedersen SS, Denollet J, Daemen J, et al. Fatigue, depressive symptoms, and hopelessness as predictors of adverse clinical events following percutaneous coronary intervention with paclitaxel-eluting stents. J Psychosom Res 2007;62:455-61.

22. Damen NL, Pelle AJ, Boersma E, et al. Reduced positive affect (anhedonia) is independently associated with 7-year mortality in patients treated with percutaneous coronary intervention: results from the research registry. Eur J Prev Cardiol 2013;20:127-34.

23. Stang A. Critical evaluation of the Newcastle-Ottawa scale for the assessment of the quality of nonrandomized studies in metaanalyses. Eur J Epidemiol 2010;25:603-5.

24. Higgins JPT, Thompson SG, Deeks JJ, et al. Measuring inconsistency in meta-analyses. BMJ 2003;327:557-60.

25. Egger M, Davey Smith G, Schneider M, et al. Bias in meta-analysis detected by a simple, graphical test. BMJ 1997;315:629-34.

26. Begg CB, Mazumdar M. Operating characteristics of a RANK correlation test for publication bias. Biometrics 1994;50:1088-101.

27. Zigmond AS, Snaith RP. The hospital anxiety and depression scale. Acta Psychiatr Scand 1983;67:361-70.

28. Barth J, Schumacher M, Herrmann-Lingen C. Depression as a risk factor for mortality in patients with coronary heart disease: a metaanalysis. Psychosom Med 2004;66:802-13. 
29. Bjelland I, Dahl AA, Haug TT, et al. The validity of the hospital anxiety and depression scale. An updated literature review. J Psychosom Res 2002;52:69-77.

30. Davidson KW, Kupfer DJ, Bigger JT, et al. Assessment and treatment of depression in patients with cardiovascular disease: National heart, lung, and blood Institute Working Group report. Psychosom Med 2006;68:645-50.

31. Thombs BD, Ziegelstein RC, Whooley MA. Optimizing detection of major depression among patients with coronary artery disease using the patient health questionnaire: data from the heart and soul study. $J$ Gen Intern Med 2008;23:2014-7.
32. Ren $\mathrm{Y}$, Yang $\mathrm{H}$, Browning $\mathrm{C}$, et al. Performance of screening tools in detecting major depressive disorder among patients with coronary heart disease: a systematic review. Med Sci Monit 2015;21:646-53.

33. Zhang P. Study of Anxiety/Depression in patients with coronary heart disease after percutaneous coronary intervention. Cell Biochem Biophys 2015;72:503-7.

34. Ravven S, Bader C, Azar A, et al. Depressive symptoms after CABG surgery: a meta-analysis. Harv Rev Psychiatry 2013;21:59-69.

35. May HT, Horne BD, Knight S, et al. The association of depression at any time to the risk of death following coronary artery disease diagnosis. Eur Heart J Qual Care Clin Outcomes 2017;3:296-302. 